\title{
Variability and constraint of vertebral formulae and proportions in colugos, tree shrews, and rodents, with special reference to vertebral modification by aerodynamic adaptation
}

\author{
T. Kawashima1, R.W. Thorington Jr. ${ }^{2}$, P.W. Bohaska², F. Sato ${ }^{1}$ \\ ${ }^{1}$ Department of Anatomy, School of Medicine, Toho University, Tokyo, Japan \\ 2Department of Vertebrae Zoology, National Museum of Natural History, Smithsonian Institution, Washington, DC, \\ United States
}

[Received: 4 April 2017; Accepted: 19 June 2017]

Background: The aim of the present study is to provide the first large data set on vertebral formulae and proportions, and examine their relationship with different locomotive modes in colugos (Dermoptera), tree shrews (Scandentia), and rodents (Rodentia), which have been considered less variable because they were thought to have a plesiomorphic number of 19 thoracolumbar vertebrae.

Materials and methods: The data included 33 colugos and 112 tree shrews, which are phylogenetically sister taxa, and 288 additional skeletons from 29 other mammalian species adapted to different locomotive modes, flying, gliding, arboreal, terrestrial, digging, and semi-aquatic habitats.

Results: The following results were obtained: (1) intra-linterspecies variability and geographical variation in thoracic, lumbar, and thoracolumbar counts were present in two gliding colugo species and 12 terrestrial/arboreal tree shrew species; (2) in our examined mammals, some aerodynamic mammals, such as colugos, southern flying squirrels, scaly-tailed squirrels, and bats, showed exceptionally high amounts of intraspecific variation of thoracic, lumbar, and thoracolumbar counts, and sugar gliders and some semi-aquatic rodents also showed some variation; (3) longer thoracic and shorter lumbar vertebrae were typically shared traits among the examined mammals, except for flying squirrels (Pteromyini) and scaly-tailed squirrels (Anomaluridae).

Conclusions: Our study reveals that aerodynamic adaptation could potentially lead to strong selection and modification of vertebral formulae and/or proportions based on locomotive mode despite evolutionary and developmental constraints. (Folia Morphol 2018; 77, 1: 44-56)

Key words: colugo, tree shrew, aerodynamic mammals, vertebral column, gliding adaptation

\section{INTRODUCTION}

Narita and Kuratani [33] provided developmental understanding of mammalian axial vertebral formulae using classical descriptive data by Owen [38]; they concluded that 19 thoracolumbar (TL) vertebrae in many mammalian groups tend to be fixed because

Address for correspondence: Dr. T. Kawashima, Department of Anatomy, School of Medicine, Toho University. 5-21-16 Omori-Nishi, Ota-ku, Tokyo 143-8540, Japan, tel: +81-3-3762-4151, fax: +81-3-5493-5411, e-mail: tomokazu.kawashima@med.toho-u.ac.jp 
of developmental constraints, as seen in a stable cervical count of 7; however, most Carnivora have 20 TL vertebrae, which shows a lineage-specific pattern. Because of the limited sample size in Owen's catalogue [38], to obtain more accurate conclusions it is necessary to examine a larger sample size, as in some studies [1, 39, 41, 54, 55, 62].

Asher et al. [2] conducted a statistical analysis on mammalian vertebral formulae using a larger sample size and concluded that xenarthrans and afrotherians have a high proportion of individuals with meristic deviation from species' median counts, and monotremes, xenarthrans, afrotherians, and primates have relatively high variation in TL count [2]. The extreme vertebral formulae in some mammals, such as afrotherians and xenarthrans, have recently been thoroughly re-examined [7, 23, 42].

Alternatively, it is well known that rodents are the least variable mammals and are thought to have a fixed TL count of 19 without any deviations [2, 42]. However, they live in a wide variety of habitats, such as air, trees, land, water, and soil; additionally, they represent $38 \%$ of mammal species [10]. To identify the lineage-specific trend in TL counts of rodents, various rodents should be examined, from frequently used experimental rodents to uniquely specialised rodents, such as gliding scaly-tailed squirrels (Anomalidae) and burrowing pocket gophers (Geomyodae), which have not been previously studied.

In our past research on colugos [27, 28], we found that there was insufficient data regarding colugo anatomy compared with other mammals. Colugos, arboreal and nocturnal mammals with a specialised ability to glide, tend to evade capture [31]. There are therefore limited specimens available for research.

We tested the hypothesis that axial vertebrae adapt to different locomotive modes, and are modified despite evolutionary and developmental constraints, using some of the perceived least variable mammals with regard to thoracolumbar count (fixed at 19), including colugos, tree shrews, and rodents.

The aims of the present study are to (1) provide the first large data set on vertebral formulae and proportions in colugos and tree shrews, which are sister taxa; (2) compare these data with those of rodents adapted to different locomotive modes, such as those that are gliding/arboreal, terrestrial, digging, or semi-aquatic, and include all five aerodynamic mammals: colugos, flying squirrels, scaly-tailed squirrels, sugar gliders, and bats; and (3) elucidate the relation- ships among axial vertebral counts and proportions, lineage-specific developmental constraints, and/or functional adaptation by different locomotive modes.

\section{MATERIALS AND METHODS}

\section{Materials used}

In total, 433 postcranial skeletons were used for this study, as listed in Table 1.

As the main materials, the vertebral formulae and proportions of 33 colugos (or flying lemurs; Dermoptera) and 112 tree shrews (Scandentia) were intensively examined from dry skeletons to assess their morphological similarities and differences, because they are phylogenetically sister taxa.

Furthermore, an additional 288 postcranial skeletons from 29 mammalian specimens of 16 rodent, bat, and marsupials (sub-) families that include all other aerodynamic mammals were compared to test the hypothesis that morphological changes of vertebral formula and proportion differ based on locomotive mode. Any individuals with pathological abnormalities were excluded from this study.

\section{Identification and measurement of vertebrae}

For identification of each vertebra, the vertebral morphology was considered the most important criterion, as in other studies $[2,40]$.

Thoracic vertebrae were principally identified as those elements with rib facets on or near the vertebral neural arch, or the presence of articulated ribs. In addition, anatomical characteristics were usually present, such as species-dependent morphology of the shape, size, width, height, and angle of the vertebral body, and transverse and spinous processes with lumbar vertebrae (Fig. 1A, B).

If there were difficulties with identification of short unarticulated ribs or long lumbar transverse processes (Fig. 1C), thoracic and lumbar vertebrae were identified based on the above anatomical characteristics; the thoracic vertebrae were generally more slender and weaker than lumbar vertebrae [40], although these vertebral characteristics differ by species.

Lumbar vertebrae were identified as those elements cranial to the sacro-iliac articulation lacking conspicuous rib facets and showing a transverse process (Fig. 1D). Some difficult cases, in which the most caudal lumbar vertebra partially fused into sacral vertebrae as one sacral bone and articulated with the iliac bone, were defined based on anatomical characteristics. 
Table 1. Examined materials

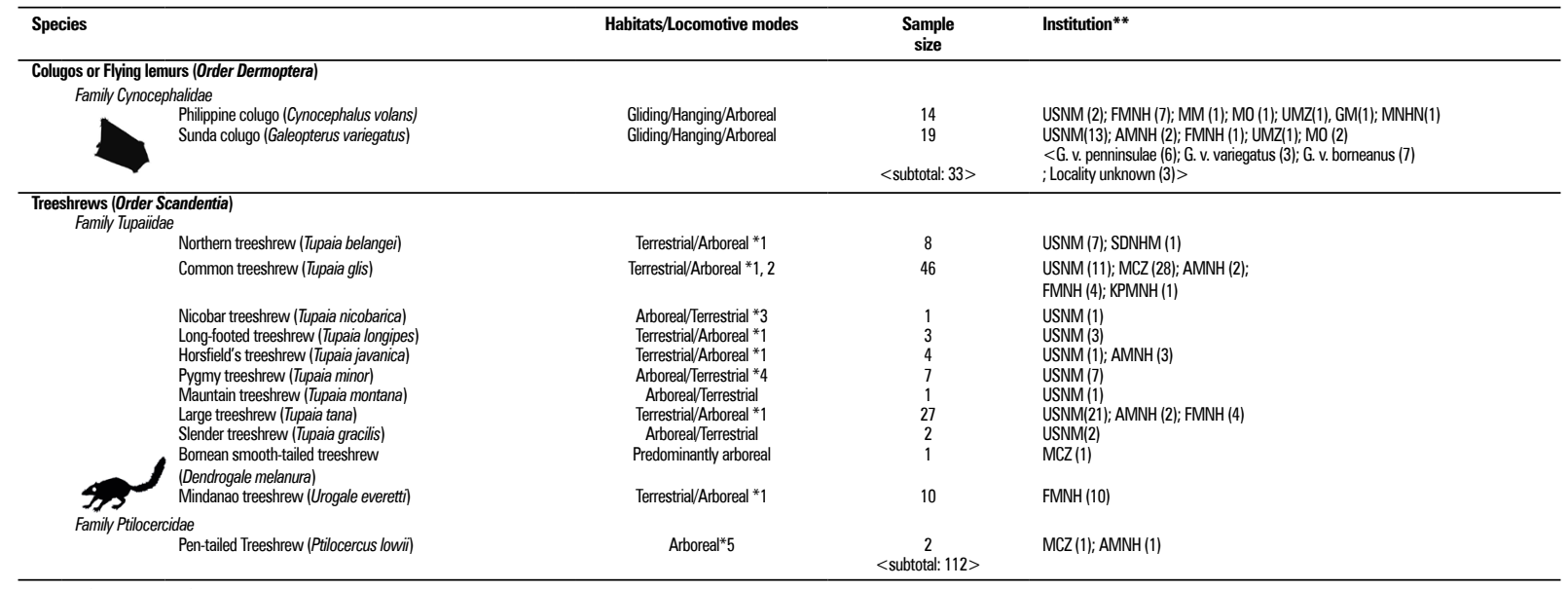

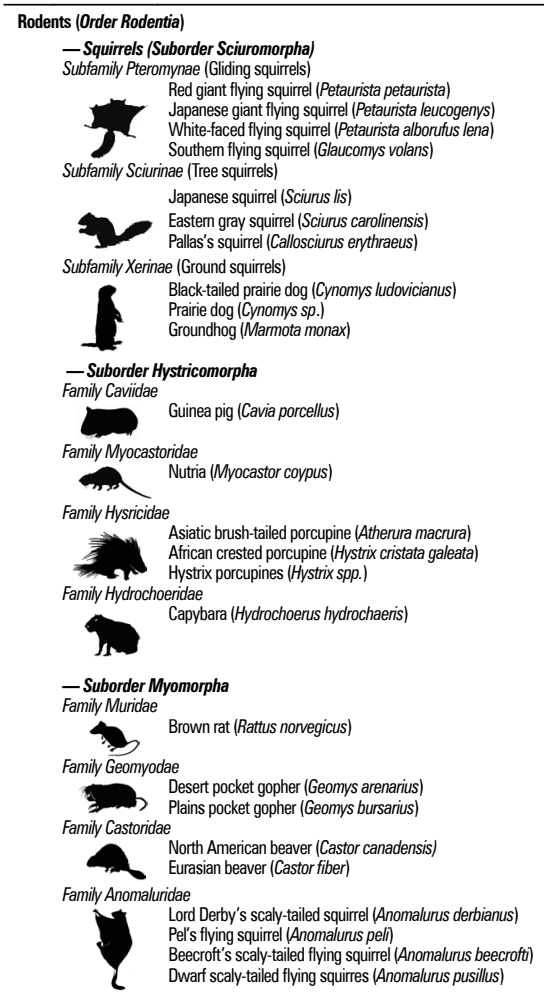

\begin{tabular}{|c|c|c|}
\hline $\begin{array}{l}\text { Gliding/Arboreal } \\
\text { Gliding/Arboreal } \\
\text { Gliding/Arboreal } \\
\text { Gliding/Arboreal }\end{array}$ & $\begin{array}{l}13 \\
12 \\
22 \\
18\end{array}$ & $\begin{array}{l}\text { USNM (10); NTU (3) } \\
\text { USNM (1); DMU (2); KPMNH (9) } \\
\text { USNM (3); TNMNS (19) } \\
\text { USNM(16); TH (2) }\end{array}$ \\
\hline Arborea//errestrial & 6 & DMU (4); KPMNH (2) \\
\hline Arborea/Terrestrial & $\begin{array}{l}16 \\
14\end{array}$ & $\begin{array}{l}\text { USNM (16) } \\
\text { TMNS (14) }\end{array}$ \\
\hline Terrestria/Digging & 7 & USNM (7) \\
\hline Terrestria/Digging & 4 & $\mathrm{DMU}(4)$ \\
\hline Terrestria/Digging & $\begin{array}{c}11 \\
<123>\end{array}$ & USNM (9); DMU (2) \\
\hline Terrestria/Digging & 11 & USNM (4); TH(3): TNMNS (1); OCMNH (3) \\
\hline Semi-aquatic & 13 & USNM (6); DMU(2); KPMNH (1); OCMNH (4) \\
\hline Terrestria/Digging & 7 & USNM (3); AMNH (4) \\
\hline Terrestria/Digging & 8 & $\begin{array}{l}\text { USNM (1); AMNH (4); DMU (1); NTUZM (1); OCMNH (1) }\end{array}$ \\
\hline Terrestria/Digging & 4 & USNM (2); NTUZM (2) \\
\hline Semi-aquatic & $\begin{array}{c}10 \\
<53>\end{array}$ & USNM (4); DMU (3); KPMNH (3) \\
\hline Terrestrial & 14 & USNM (10); TWMU (2); TH (1); DMU(1) \\
\hline $\begin{array}{l}\text { Terrestria/Digging } \\
\text { Terrestria/Digging }\end{array}$ & $\begin{array}{l}3 \\
9\end{array}$ & $\begin{array}{l}\text { USNM (3) } \\
\text { USNM (9) }\end{array}$ \\
\hline Semi-aquatic & 13 & USNM (12); DMU (1) \\
\hline Semi-aquatic & 2 & USNM (2) \\
\hline $\begin{array}{l}\text { Gliding/Arboreal } \\
\text { Gliding/Arboreal } \\
\text { Gliding Arboreal } \\
\text { Gliding/Arboreal }\end{array}$ & $\begin{array}{c}13 \\
2 \\
2 \\
2 \\
<60>\end{array}$ & $\begin{array}{l}\text { USNM (5); AMNH (8) } \\
\text { USNM (2) } \\
\text { USNM (1); AMNH (1) } \\
\text { AMNH (2) }\end{array}$ \\
\hline
\end{tabular}

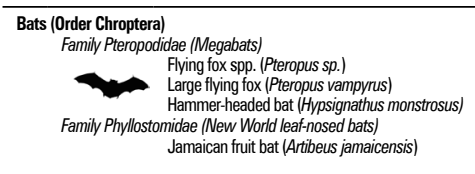

$<$ subtotal: $236>$

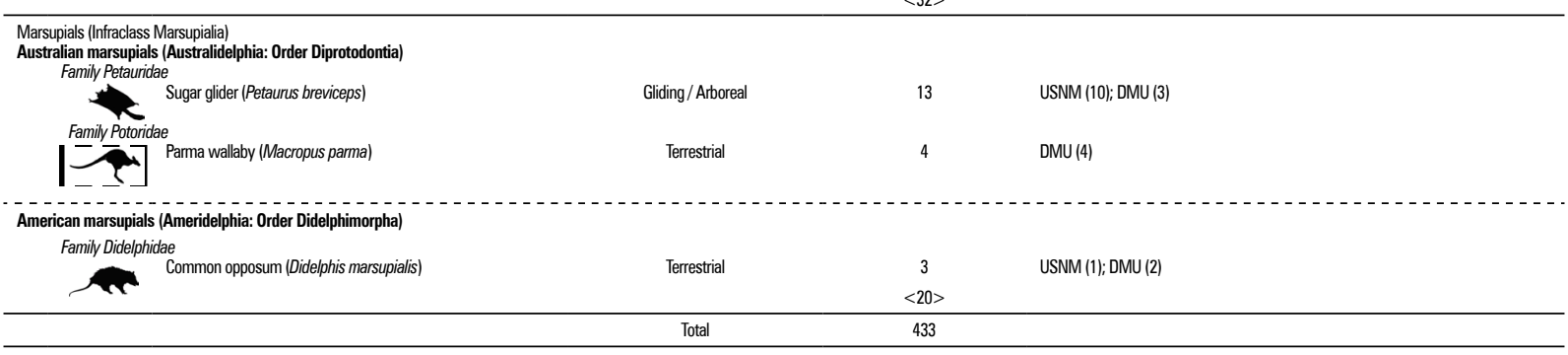

${ }_{* 1}^{*}$ most tupaiies, more terrestrial (Wharton, 1950)

" ${ }^{* 2}$ more teresestrial, Nowak, R. (1999). Walker's Mammals of the Wordd (6th Ed.) Vol 1. Battimore and London: The JohnsHopkins University Press. pp.245-246.

Prashanth \& Veenahumari, 1996)

*4 T. minor, arboreal (Lim, 1969; D'SSOuza, 1974)
${ }_{*}^{*}$ P Ptilocerus, arboreal (Lim, 1967, 1969; Muul \& Lim, 1971; Gould, 1978)" "**institutions: AMNH, American Museum of Natural History (New York, NY, USA); DMU, Dokkyo Medical University (Mibu, Japan); FMNH, Field Museum of Natural History (Chicago, LL, USA); GM, Grant Museum of Zoology and Comparative Anatomy at University College of London (London, UK); KPMNH, Kanagawa Prefectural Museum of Natural History (Odawara, Japan); MCZ, Museum of Comparative Zoology at Havard University (Boston, MA, USA); MM, Melbourne Museum (Melboume, Australia; MNHN, Museum National

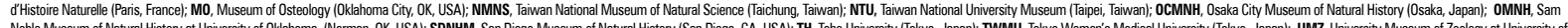

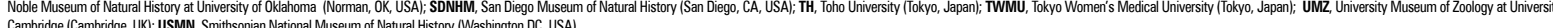


All vertebral measurements were taken based on the ventral height of each vertebral body using a digital calliper.

\section{RESULTS}

\section{Vertebral formula}

The vertebral formulae and TL counts are shown in Tables 2 and 3, respectively. Our findings showed that the range in vertebral formulae were $7 \mathrm{C}$ (cervical vertebrae), 13-14T (thoracic vertebrae), 5-8L (lumbar vertebrae), 4-5S (sacral vertebrae), and 18-22TL (thoracolumbar vertebrae) in colugos and 7C, 12-15T, 5-7L, $3 S$, and 18-21TL in tree shrews. Although the most frequent formula of presacral vertebrae was shared as 7C, 13T, 6L, and 19TL in both species (Tables 2, 3), our data showed that the most frequent TL count of 19 was found in only $48.5 \%$ of colugos (16/33) and $72.3 \%$ of tree shrews (81/112). Furthermore, geographical variation was also observed: the most frequent TL counts were 21 or 22 (each $35.7 \%, 5 / 14$ ) in Philippine colugos and 19 (68.4\%: 13/19) in Sunda colugos (Fig. 2).

In examined rodents, all tree and most ground squirrels, Guinea pigs, porcupines, rats, and pocket gophers, had consistently fixed TL counts of 19 (100.0\%; Fig. 3A). Some rodents showed variation from 19 TL counts: black-tailed prairie dog, 6/7 cases $(85.7 \%)$; nutria, $11 / 13$ (84.6\%); capybara, $8 / 10$ (80.0\%); North American beavers, 11/13 (84.6\%); red giant flying squirrel, 9/13 (75.0\%); Japanese giant flying squirrel, 10/12 (83.3\%); and southern flying squirrel, 14/18 (77.8\%). In addition, scaly-tailed squirrels exhibited extreme TL counts of 24 (1/19, 5.3\%), $25(17 / 19,89.5 \%)$, and $26(1 / 19,5.3 \%)$.

In bats, TL variability was also observed: Pteropus megabats, 17TL $(2 / 21,9.5 \%)$ and 18TL (19/21, 90.5\%); and Jamaican fruit bats, $16 \mathrm{TL}(1 / 11,9.1 \%)$, 17TL (9/11, 81.6\%), and 18TL (1/11, 9.1\%).

In examined marsupials, all Parma wallabies and common opossums had fixed TL counts of 19 $(100.0 \%)$, but the sugar gliders had some variation: $18 \mathrm{TL}(2 / 13,15.4 \%)$ and $19 \mathrm{TL}(11 / 13,84.6 \%)$.

These results showed that variability of TL counts in range and frequency predominated in most aerodynamic mammals and there was variation from 19TL in some semi-aquatic mammals.

\section{Relative lengths of axial skeletons}

To eliminate a bias caused by different vertebral formulae, only axial skeletons with the most frequent vertebral formula for each species were selected; then, lengths of
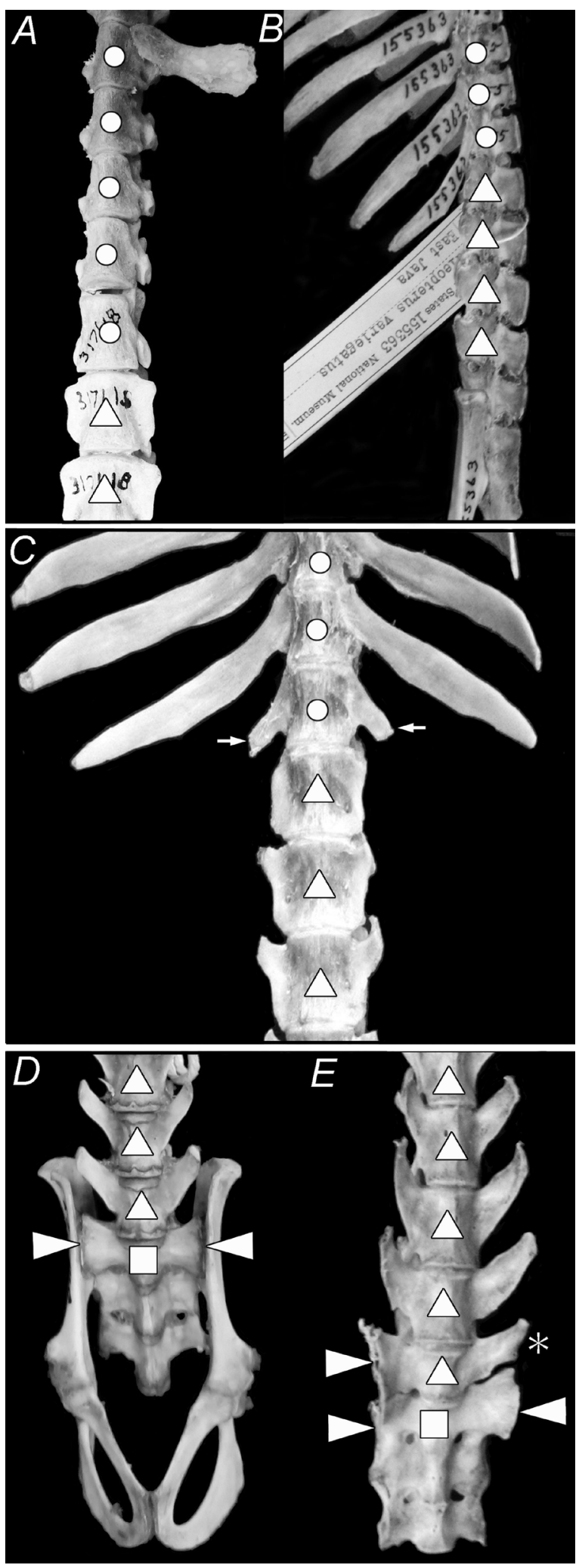

Figure 1. Vertebral identification. Circles $(\bullet)$, triangles $(\boldsymbol{\Delta})$, and squares ( $\square$ ) show the thoracic, lumbar, and sacral vertebrae, respectively; A-C. Thoracic and lumbar differences. A difficult case such as an unarticulated lumbar rib, as shown with arrows, is classified based on morphology and size of vertebra; D-E. Lumbar and sacral differences. Arrowheads show the sacroiliac articulation. 
Table 2. Variation of thoracic $(T)$, lumbar (L), and sacral $(S)$ vertebral counts

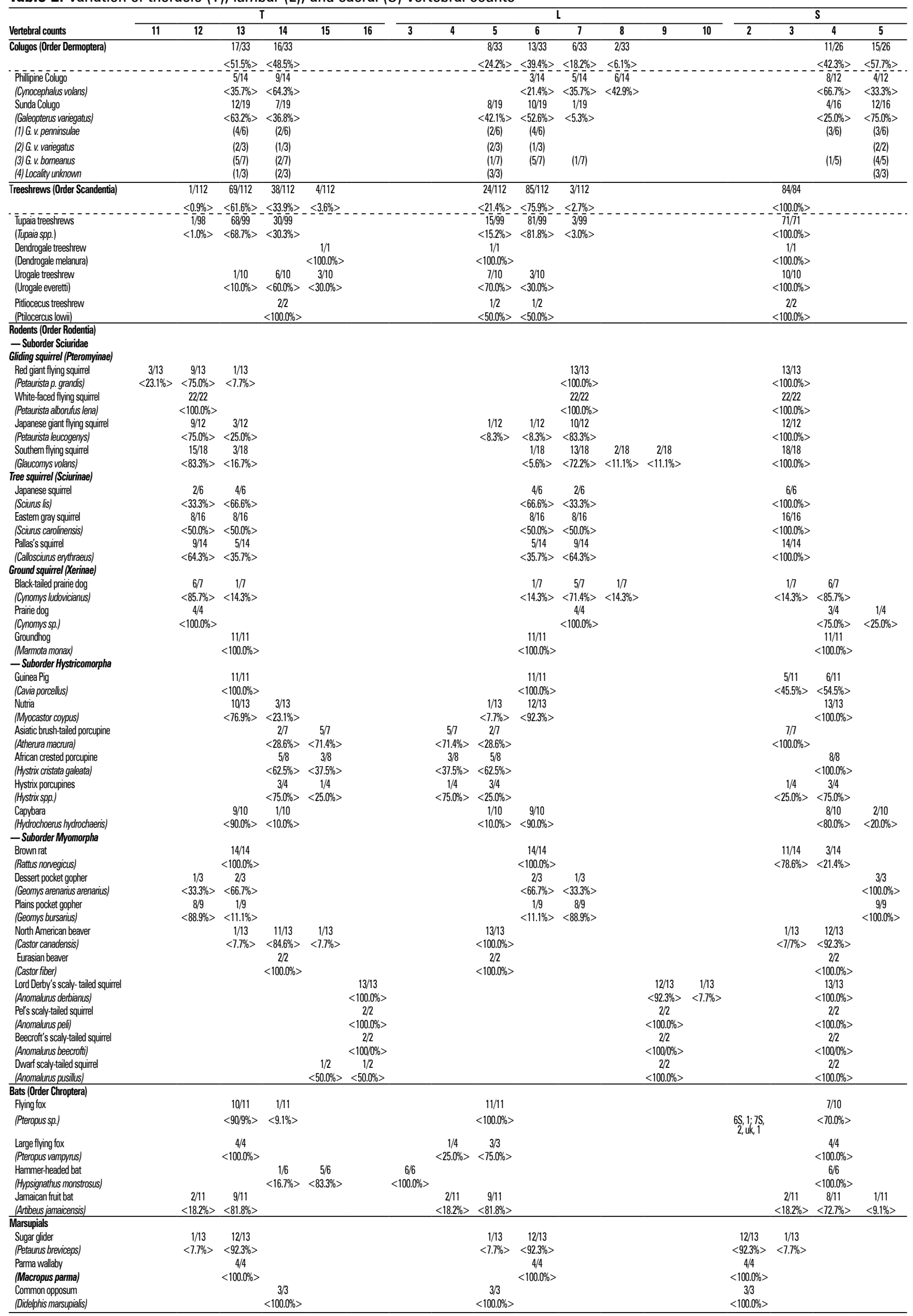


Table 3. Variation of thoracolumbar (TL) vertebral counts

\begin{tabular}{|c|c|c|c|c|c|c|c|c|c|c|c|}
\hline Number of Vertebrae & 16 & 17 & 18 & 19 & 20 & 21 & 22 & 24 & 25 & 26 & \\
\hline \multirow[t]{2}{*}{ Colugos (Order Dermoptera) } & & & $3 / 33(9.1 \%)$ & $16 / 33(48.5 \%)$ & $4 / 33(12.1 \%)$ & $5 / 33(15.2 \%)$ & $5 / 33(15.2 \%)$ & & & & \\
\hline & & & & & & & & & & & $100 \%$ \\
\hline \multirow[t]{2}{*}{ Phillipine Colugo (Cynocephalus volans) } & & & & $3 / 14(21.4 \%)$ & $\overline{1} / \overline{14}(\overline{7} . \overline{1})$ & $5 / 14(35.7 \%)$ & $5 / 14(35.7 \%)$ & & & & $50-99 \%$ \\
\hline & & & & & & & & & & & $10-49 \%$ \\
\hline \multicolumn{3}{|l|}{ Sunda Colugo (Galeopterus variegatus) } & $3 / 19(15.8 \%)$ & $13 / 19(68.4 \%)$ & $3 / 19(15.8 \%)$ & & & & & & $<10 \%$ \\
\hline \multicolumn{3}{|l|}{ (1) G. v. penninsulae } & $(1 / 5)$ & $(4 / 6)$ & $(1 / 5)$ & & & & & & \\
\hline \multicolumn{3}{|l|}{ (2) G. v. variegatus } & $(1 / 3)$ & $(2 / 3)$ & & & & & & & \\
\hline \multicolumn{3}{|l|}{ (3) G. v. bomeanus } & & $(5 / 7)$ & $(2 / 7)$ & & & & & & \\
\hline \multicolumn{3}{|l|}{ (4) Locality unknown } & $(1 / 3)$ & $(2 / 3)$ & & & & & & & \\
\hline \multicolumn{3}{|l|}{ Treeshrews (Order Scandentia) } & $3 / 112(2.7 \%)$ & $81 / 112(72.3 \%)$ & $26 / 112(23.2 \%)$ & $2 / 112(1.8 \%)$ & & & & & \\
\hline \multicolumn{3}{|l|}{ Tupaia tree shrews (Tupaia spp.) } & $3 / 99(3.0 \%)$ & $75 / 99(75.8 \%)$ & $21 / 99(21.2 \%)$ & 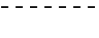 & & & & & \\
\hline \multicolumn{3}{|l|}{ Bomean smooth-tailed tree shrew (Dendrogale melanura) } & & & $1 / 1(100 \%)$ & & & & & & \\
\hline \multicolumn{3}{|l|}{ Mindanao tree shrew (Urogale everetti) } & & $5 / 10(50.0 \%)$ & $3 / 10(30.0 \%)$ & $2 / 10(20.0 \%)$ & & & & & \\
\hline \multicolumn{3}{|l|}{ Pen-tailed tree shrew (Ptilocercus lowii) } & & $1 / 2(50.0 \%)$ & $1 / 2(50.0 \%)$ & & & & & & \\
\hline \multicolumn{12}{|l|}{ <0ther mammals $>$} \\
\hline \multicolumn{12}{|l|}{ Squirrels (Suborder Sciuridae) } \\
\hline \multicolumn{12}{|l|}{ Gliding squirrel (Pteromyinae) } \\
\hline Red giant flying squirrel (Petaurista p. grandis) & & & $3 / 13(23.1 \%)$ & 9/13(75.0\%) & & & & & & & \\
\hline White-faced flying squirrel (Petaurista alborufus lena) & & & & $22 / 22(100.0 \%)$ & & & & & & & \\
\hline Japanese giant flying squirrel (Petaurista leucogenys) & & & 1/12 (8.3\%) & 10/12 (83.3\%) & $1 / 12(8.3 \%)$ & & & & & & \\
\hline Southem flying squirrel (Glaucomys volans) & & & & $14 / 18(77.8 \%)$ & $2 / 18(11.1 \%)$ & $2 / 18(11.1 \%)$ & & & & & \\
\hline Tree squirrel (Sciurinae) & & & & & & & & & & & \\
\hline Japanese squirrel (Sciurus lis) & & & & $6 / 6(100.0 \%)$ & & & & & & & \\
\hline Eastern gray squirrel (Sciurus carolinensis) & & & & $16 / 16(100.0 \%)$ & & & & & & & \\
\hline Pallas's squirrel (Callosciurus erythraeus) & & & & $14 / 14(100.0 \%)$ & & & & & & & \\
\hline Ground squirrel (Xerinae) & & & & & & & & & & & \\
\hline Black-tailed prairie dog (Cynomys /udovicianus) & & & & $6 / 7(85.7 \%)$ & $1 / 7(14.3 \%)$ & & & & & & \\
\hline Prairie dog (Cynomys sp.) & & & & $4 / 4(100.0 \%)$ & & & & & & & \\
\hline Groundhog (Marmota monax) & & & & 11/11(100.0\%) & & & & & & & \\
\hline Suborder Hystricomorpha & & & & & & & & & & & \\
\hline Guinea Pig (Cavia porcellus) & & & & $11 / 11(100.0 \%)$ & & & & & & & \\
\hline Nutria (Myocastor coypus) & & & & $11 / 13(84.6 \%)$ & $2 / 13(15.4 \%)$ & & & & & & \\
\hline Asiatic brush-tailed porcupine (Atherura macrura) & & & & $7 / 7(100.0 \%)$ & & & & & & & \\
\hline African crested porcupine (Hystrix cristata galeata) & & & & $8 / 8(100.0 \%)$ & & & & & & & \\
\hline Hystrix porcupines (Hystrix sp.) & & & & $4 / 4(100.0 \%)$ & & & & & & & \\
\hline Capybara (Hydrochoerus hydrochaeris) & & & $1 / 10(10.0 \%)$ & $8 / 10(80.0 \%)$ & $1 / 10(10.0 \%)$ & & & & & & \\
\hline Suborder Myomorpha & & & & & & & & & & & \\
\hline Brown rat (Rattus norvegicus) & & & & 14/14 (100.0\%) & & & & & & & \\
\hline Desert pocket gopher (Geomys arenarius arenarius) & & & & $3 / 3(100.0 \%)$ & & & & & & & \\
\hline Plains pocket gopher (Geomys bursarius) & & & & 9/9 (100.0\%) & & & & & & & \\
\hline North American beaver (Castor canadensis) & & & $1 / 13(7.7 \%)$ & $11 / 13(84.6 \%)$ & $1 / 13(7.7 \%)$ & & & & & & \\
\hline Eurasian beaver (Castor fiber) & & & & $2 / 2(100.0 \%)$ & & & & & & & \\
\hline Lord Derby's scaly-tailed squirrel (Anomalurus derbianus) & & & & & & & & & $12 / 13(92.3 \%)$ & $1 / 13(7.7 \%)$ & \\
\hline Pel's scaly-tailed squirrel (Anomalurus peli) & & & & & & & & & $2 / 2(100.0 \%)$ & & \\
\hline Beecroft's scaly-tailed squirrel (Anomalurus beecrofti) & & & & & & & & & $2 / 2(100.0 \%)$ & & \\
\hline Dwarf scaly-tailed squirrel (Anomalurus pusillus) & & & & & & & & $2(50.0 \%)$ & $1 / 2(50.0 \%)$ & & \\
\hline Bats (Order Chroptera) & & & & & & & & & & & \\
\hline Megabat (Pteropodidae) & & & & & & & & & & & \\
\hline Flying fox (Pteropus sp.) & & & 10/11 (90.9\%) & 1/11(9.1\%) & & & & & & & \\
\hline Large flying fox (Pteropus vampyrus) & & $1 / 4(25.0 \%)$ & $3 / 4(75.0 \%)$ & & & & & & & & \\
\hline Hammer-headed bat (Hypsignathus monstrosus) & & $1 / 6(16.7 \%)$ & $5 / 6(83.3 \%)$ & & & & & & & & \\
\hline $\begin{array}{l}\text { New World leaf-nosed bat (Phylllostomidae) } \\
\text { Jamaican fruit bat (Artibeus jamaicensis) }\end{array}$ & $11(9.1$ & $9 / 11(81.6 \%)$ & $1 / 11(9.1 \%)$ & & & & & & & & \\
\hline Marsupials & & & & & & & & & & & \\
\hline Sugar glider (Petaurus breviceps) & & & $2 / 13(15.4 \%)$ & $11 / 13(84.6 \%)$ & & & & & & & \\
\hline Parma wallaby (Macropus parma) & & & & $4 / 4(100.0 \%)$ & & & & & & & \\
\hline Common opposum (Didelphis marsupialis) & & & & $3 / 3(100.0 \%)$ & & & & & & & \\
\hline
\end{tabular}

cervical, thoracic, lumbar, and sacral vertebrae relative to body length (TL length) were calculated as summarised in Table 4. Most examined mammals except flying squirrels [Pteromynae: giant (Fig. 3B) and southern (Fig. 3C) flying squirrels] and scaly-tailed squirrels (Anomalidae: Fig. 3E, F) had longer thoracic and shorter lumbar vertebrae.

Remarkably, two aerodynamic mammals, colugos and bats, had elongated cervical vertebrae (colugo: Fig. 1A, C, E; bat, Fig. 3G).
Consequently, unique elongations in relative axial length (thoracic and lumbar vertebrae) were recognised as aerodynamic mammalian characteristics.

\section{DISCUSSION}

\section{Functional adaptation and developmental} constraints of vertebral formulae

To date, developmental studies clarified that specification of the vertebral column is regulated by the 
Table 4. Relative lengths of each vertebrae to body length (thoracolumbar length) in most frequent vertebral formula

\begin{tabular}{|c|c|c|c|c|c|c|c|}
\hline Common name (scientific name) & $\begin{array}{l}\text { Selected vertebral } \\
\text { formula }\end{array}$ & $\begin{array}{l}\text { Sample } \\
\text { size }\end{array}$ & Stage & C & $\mathbf{T}$ & $\mathbf{L}$ & S \\
\hline \multicolumn{8}{|l|}{ Gliding / Flying } \\
\hline \multirow[t]{3}{*}{ Sunda colugo (Geleopterus variegatus) } & 7C13T6L5S (19TL) & 9 & Total: 9 & $39.3 \pm 6.1 \%$ & $61.8 \pm 1.4 \%$ & $38.2 \pm 1.4 \%$ & $25.3 \pm 3.6 \%$ \\
\hline & & & (Adult: 7) & $36.9 \pm 4.3 \%$ & $61.7 \pm 1.0 \%$ & $38.3 \pm 1.0 \%$ & $26.4 \pm 3.4 \%$ \\
\hline & & & (Juvenile: 2) & $47.7 \pm 2.2 \%$ & $62.3 \pm 2.9 \%$ & $37.7 \pm 2.9 \%$ & $21.6 \pm 1.3 \%$ \\
\hline Red giant flying squirrel (Petaurista petaurista) & 7C12T7L3S (19TL) & 9 & Adult: 9 & $15.6 \pm 1.8 \%$ & $44.5 \pm 0.8 \%$ & $55.5 \pm 0.8 \%$ & $14.9 \pm 1.4 \%$ \\
\hline White-faced flying squirrel (Petaurista alborufus) & 7C12T7L3S (19TL) & 22 & Adult: 22 & $15.2 \pm 1.1 \%$ & $46.0 \pm 2.3 \%$ & $54.0 \pm 2.3 \%$ & $15.3 \pm 1.3 \%$ \\
\hline Japanese giant flying squirrel (Petaurista leucogenys) & 7C12T7L3S (19TL) & 8 & Adult: 8 & $16.9 \pm 0.9 \%$ & $44.2 \pm 1.4 \%$ & $55.8 \pm 1.4 \%$ & $16.3 \pm 1.4 \%$ \\
\hline \multirow[t]{3}{*}{ Southern flying squirrel (Glaucomys volans) } & 7C12T7L3S (19TL) & 12 & Total: 12 & $18.5 \pm 3.2 \%$ & $47.4 \pm 1.7 \%$ & $52.6 \pm 1.7 \%$ & $17.5 \pm 4.1 \%$ \\
\hline & & & (Adult: 9) & $17.5 \pm 2.3 \%$ & $47.4 \pm 1.3 \%$ & $52.6 \pm 1.3 \%$ & $15.4 \pm 2.0 \%$ \\
\hline & & & (Juvenile: 3 ) & $22.5 \pm 1.5 \%$ & $46.4 \pm 2.7 \%$ & $53.6 \pm 2.7 \%$ & $22.8 \pm 3.2 \%$ \\
\hline \multirow[t]{3}{*}{ Lord Derby's scaly-tailed squirrels (Anomalurus derbianus) } & 7C16T9L4S (25TL) & 10 & Total: 10 & $18.5 \pm 1.1 \%$ & $48.6 \pm 1.0 \%$ & $51.4 \pm 1.0 \%$ & $17.2 \pm 1.1 \%$ \\
\hline & & & (Adult: 9) & $18.6 \pm 1.1 \%$ & $48.5 \pm 1.0 \%$ & $51.5 \pm 1.0 \%$ & $16.9 \pm 0.6 \%$ \\
\hline & & & (Juvenile: 1) & $16,90 \%$ & $49,60 \%$ & $50.4 \%$ & $19,80 \%$ \\
\hline Sugar glider (Petaurus breviceps) & 7C13T6L2S (19TL) & 10 & Adult: 10 & $17.1 \pm 1.1 \%$ & $53.4 \pm 1.8 \%$ & $46.7 \pm 1.8 \%$ & $10.0 \pm 1.0 \%$ \\
\hline Megabats (Pteropus spp.) & 7C13T5L4S (18TL) & 7 & Adult: 7 & $48.1 \pm 4.0 \%$ & $68.1 \pm 2.3 \%$ & $31.9 \pm 2.3 \%$ & $23.5 \pm 2.0 \%$ \\
\hline Large flying fox (Pteropus vampyrus) & 7C13T5L4S (18TL) & 3 & Adult: 3 & $40.5 \pm 2.1 \%$ & $69.0 \pm 2.1 \%$ & $31.0 \pm 2.1 \%$ & $22.1 \pm 4.1 \%$ \\
\hline Jamaican fruit bat (Artibeus jamaicensis) & 7C13T4L4S (17TL) & 5 & Adult:5 & $24.1 \pm 5.8 \%$ & $74.1 \pm 3.5 \%$ & $25.9 \pm 3.5 \%$ & $22.9 \pm 3.9 \%$ \\
\hline Hammer-headed bat (Hypsignathus monstrosus) & 7C15T3L4S (18TL) & 5 & Adult:5 & $37.7 \pm 4.1 \%$ & $81.3 \pm 0.4 \%$ & $18.7 \pm 0.4 \%$ & $16.2 \pm 1.0 \%$ \\
\hline \multicolumn{8}{|l|}{ Arboreal/Terrestrial } \\
\hline Common tree shrew (Tupaia glis) & 7C13T6L3S (19TL) & 6 & Adult: 6 & $18.5 \pm 1.8 \%$ & $53.6 \pm 3.2 \%$ & $46.5 \pm 3.2 \%$ & $14.3 \pm 1.2 \%$ \\
\hline Japanese squirrel (Sciurus lis) & 7C13T6L3S (19TL) & 4 & Adult: 4 & $19.4 \pm 1.8 \%$ & $56.5 \pm 1.8 \%$ & $43.5 \pm 1.8 \%$ & $16.7 \pm 1.1 \%$ \\
\hline Eastern Gray squirrel (Sciurus carolinensis) & 7C13T6L3S (19TL) & 8 & Adult: 8 & $17.9 \pm 1.0 \%$ & $56.2 \pm 4.0 \%$ & $43.8 \pm 4.0 \%$ & $14.3 \pm 2.7 \%$ \\
\hline Pallas's squirrel (Callosciurus erythraeus) & 7C12T7L3S (19TL) & 9 & Adult: 9 & $18.3 \pm 2.5 \%$ & $52.0 \pm 2.1 \%$ & $49.6 \pm 2.1 \%$ & $14.6 \pm 1.9 \%$ \\
\hline \multicolumn{8}{|l|}{ Terrestrial/Digging } \\
\hline Rat (Rattus norvegicus) & 7C13T6L3S (19TL) & 10 & Adult: 10 & $22.7 \pm 2.3 \%$ & $55.9 \pm 3.2 \%$ & $44.1 \pm 3.2 \%$ & $23.2 \pm 5.5 \%$ \\
\hline Black-tailed prairie dog (Cynomys ludovicianus) & 7C12T7L4S (19TL) & 4 & Adult: 4 & $20.4 \pm 1.2 \%$ & $51.6 \pm 0.8 \%$ & $48.4 \pm 0.8 \%$ & $18.0 \pm 0.8 \%$ \\
\hline Groundhog (Marmota monax) & 7C13T6L4S (19TL) & 10 & Adult: 10 & $21.1 \pm 2.0 \%$ & $59.4 \pm 1.9 \%$ & $40.6 \pm 1.9 \%$ & $18.3 \pm 2.2 \%$ \\
\hline Guinea pig (Cavia porcellus) & 7C13T6L3S (19TL) & 5 & Adult: 5 & $27.1 \pm 3.2 \%$ & $67.6 \pm 3.1 \%$ & $32.4 \pm 3.1 \%$ & $20.5 \pm 2.1 \%$ \\
\hline Plains pocket gopher (Geomys bursarius) & 7C12T7L5S (19TL) & 6 & Adult: 6 & $19.6 \pm 2.0 \%$ & $51.8 \pm 2.4 \%$ & $48.2 \pm 2.4 \%$ & $31.2 \pm 4.0 \%$ \\
\hline Asiatic brush-tailed porcupine (Atherura macrura) & 7C15T4L3S (19TL) & 5 & Adult: 5 & $27.5 \pm 1.3 \%$ & $64.3 \pm 3.2 \%$ & $35.7 \pm 3.2 \%$ & $18.7 \pm 0.9 \%$ \\
\hline African crested porcupine (Hystrix cristata galeata) & 7C14T5L4S (19TL) & 5 & Adult: 5 & $29.4 \pm 2.6 \%$ & $64.2 \pm 2.0 \%$ & $35.8 \pm 2.0 \%$ & $24.5 \pm 2.9 \%$ \\
\hline \multicolumn{8}{|l|}{ Semi-aquatic } \\
\hline \multirow[t]{3}{*}{ Capybara (Hydrochoerus hydrochaeris) } & 7C13T6L4S (19TL) & 7 & Total: 7 & $32.4 \pm 6.1 \%$ & $58.8 \pm 0.9 \%$ & $48.2 \pm 0.9 \%$ & $24.1 \pm 3.3 \%$ \\
\hline & & & (Adult: 4) & $29.8 \pm 0.6 \%$ & $59.2 \pm 1.9 \%$ & $40.8 \pm 1.9 \%$ & $23.0 \pm 1.2 \%$ \\
\hline & & & (Juvenile: 3 ) & $35.8 \pm 9.0 \%$ & $58.4 \pm 0.1 \%$ & $41.6 \pm 0.1 \%$ & $25.6 \pm 4.9 \%$ \\
\hline Nutria (Myocastor coypus) & 7C13T6L4S (19TL) & 9 & Adult: 9 & $23.8 \pm 1.9 \%$ & $56.4 \pm 2.2 \%$ & $43.6 \pm 2.2 \%$ & $24.0 \pm 4.2 \%$ \\
\hline North American beaver (Castor canadensis) & 7C14T5L4S (19TL) & 11 & Adult: 11 & $18.3 \pm 1.1 \%$ & $65.2 \pm 2.5 \%$ & $34.8 \pm 2.5 \%$ & $27.6 \pm 1.6 \%$ \\
\hline
\end{tabular}

$\mathrm{C}$ - cervival; $\mathrm{T}$ - thoracic; $\mathrm{L}$ - lunbar; $\mathrm{S}$ - sacral

expression patterns of the Hox genes $[8,11,12,19]$. Additionally, somite segmentation genes also play an important role in vertebral column development [12, 24]. Based on these recent developments, TL counts were determined to be relatively constant at the levels of families and orders [33, 42]. As mentioned in those studies, many mammalian TL counts are fixed at 19, with a constant 7 cervical vertebrae and therefore, other exceptional unique mammalian species have been fo- cused to examine their evolutionary and developmental transformations in afrotherian mammals [42], sloths $[7,23,56]$, pygmy right whale [6], manatees [56], anthropoid primates [62], and dolphins [59]. Because the TL counts in colugos, tree shrews, and rodents were mostly thought to be fixed at 19 , they were excluded from detailed examination $[2,16,18,33,42]$. However, we recognised exceptions in some rodents and needed to search any selection factor for vertebral variability. 

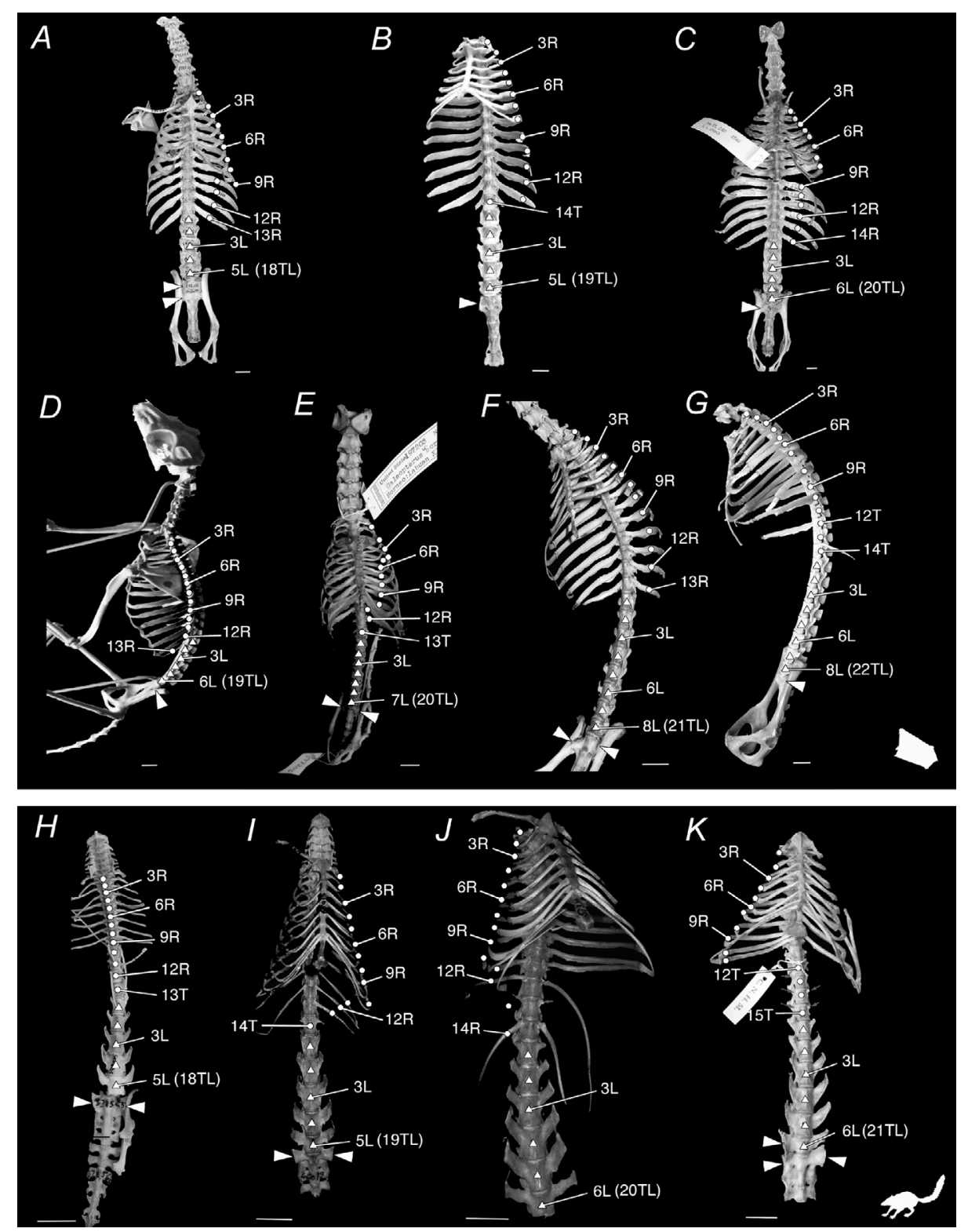

Figure 2. Morphological variability in vertebral formula for colugos $(\mathbf{A}-\mathbf{G})$ and tree shrews $(\mathbf{H}-\mathbf{K})$. Circles (৫), triangles $(\mathbf{\Delta})$, and arrowheads show the thoracic vertebrae, lumbar vertebrae, and sacroiliac articulation, respectively; A. Sunda colugo (G. variegatus), 18 TL (USNM155363);

B. Sunda colugo (G. variegatus), 19 TL (AMNH101679);

C. Sunda colugo (G. variegatus), 20 TL (USNM-A49693);

D. Philippine colugo (C. volans), 19TL (FMNH15593);

E. Philippine colugo (C. volans), 20TL (USNM197203);

F. Philippine colugo

(C. volans), 21TL (FM61031);

G. Philippine colugo (C. volans), 22TL (FMNH56438);

H. Slender tree shrew (T. gracilis), 18TL (USNM578565);

I. Common tree shrew (T. glis), 19TL (FMNH66019);

J. Mindanao tree shrew (U. everetti), 20TL (FMNH57311); K. Mindanao tree shrew (U. everetti), 21TL (FMNH61419). Scale bar $=10 \mathrm{~mm}$.

Alternatively, the vertebral counts and/or morphological transformations that reflect adaptation have been reported: elongated lumbar vertebrae in gliding squirrels compared with non-gliding squirrels contribute to increased stiffness and are useful for stability and control for gliding [54, 55]; and arboreal tree shrews are more thoracic for increased stability on trees, terrestrial tree shrews are more lumbar for increased flexibility [40]. Additionally, there are morphological differences of vertebrae in bridging and cantilevering mammals [21], and vertebral morphology and counts differ based on running speed in mammals [18].

Our previous anatomical studies on gliding mammals $[27,28]$ revealed that there were insufficient data available on axial skeleton of colugos, tree shrews, and specialised rodents, such as scaly-tailed squirrels and pocket gophers. Therefore, we investigated morphological variability of vertebral counts and proportions based on different locomotive modes.

In colugos, Leche [29] introduced some classical data on vertebral formula, but only described a few specimens, mostly one or two specimens. Although these data were clearly insufficient for comparison with other mammals, these data also seem to show vertebral variability: 7C, 13-14T, 5-9L, 3-6S and 18-22TV. In a recent study on mammalian TL counts, Sánchez-Villagra et al. [42] included 16 Philippine colugos (Cynocephalus volans) and described 19 TL 

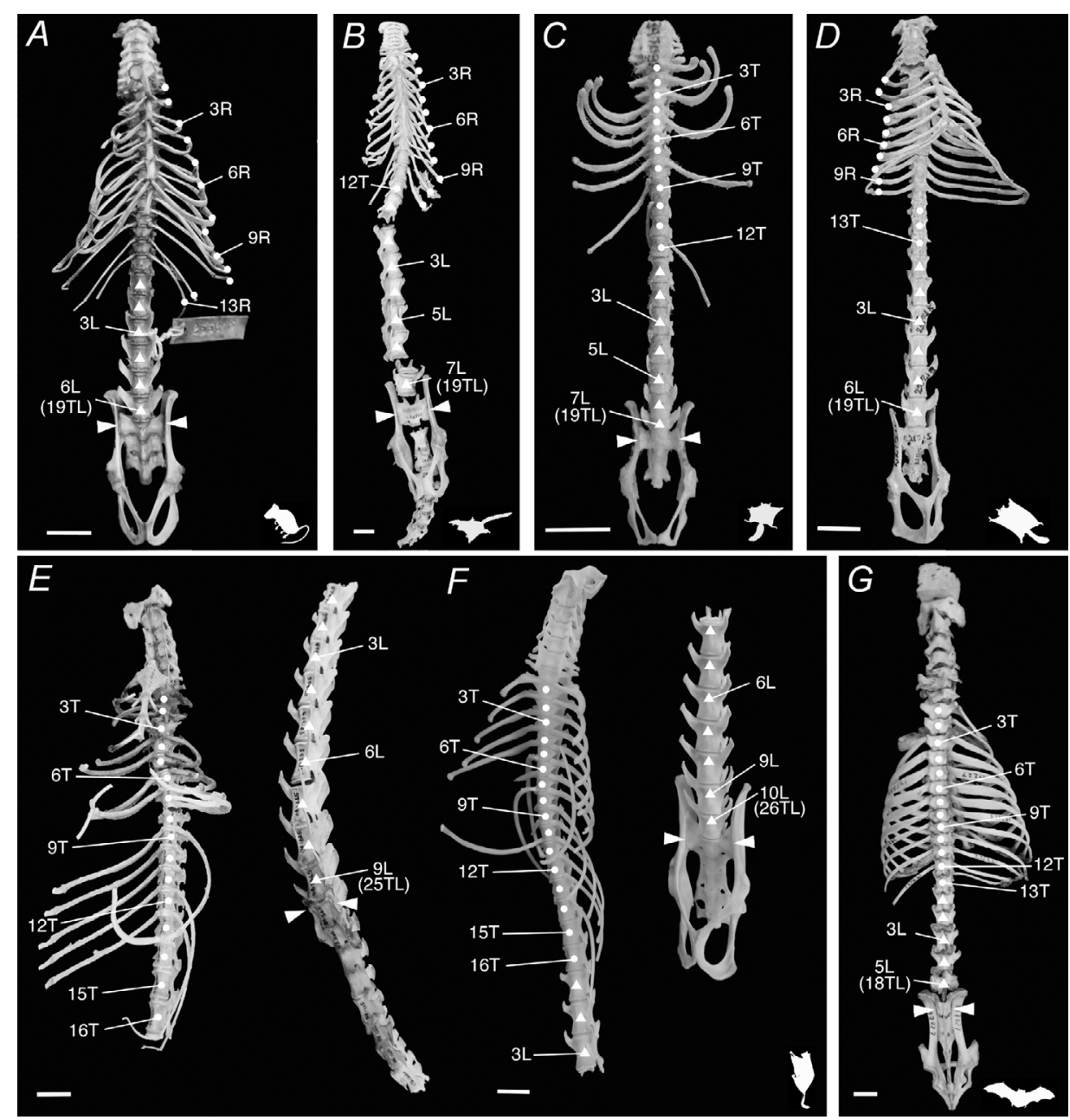

Figure 3. Comparison of vertebral formulae and proportions in a typical non-gliding rodent $(\mathbf{A})$, gliding rodents $(\mathbf{B}-\mathbf{F})$, and a flying bat (G). Circles (๑), triangles $(\boldsymbol{\Delta})$, and arrowheads show the thoracic vertebrae, lumbar vertebrae, and sacroiliac articulation, respectively. A. Typical thoracolumbar formula and proportion in most rodents, rat (19TL, USNM255345);

B. Longer lumbar vertebrae in white-faced flying squirrels (Petaurista alborufus, 19TL, USNM332937);

C. Longer lumbar vertebrae in southern flying squirrels (Glaucomys volans, 19TL, USNM569025); D. Variation on an additional lumbar vertebra with typical thoracic and sacral formula in a sugar glider (Petaurus breviceps, 19TL, USNM221215);

E, F. Constant additional and longer lumbar vertebrae in scaly-tailed squirrels: E. Anomlidae sp., 25TL, USNM599180; F. Anomalurus derbianus, 26TL, AMNH150446; G. Constant fewer lumbar vertebrae with longer cervical vertebrae in bats (Pteropus vampyrus 18TL (USNM197227). in $15(93.8 \%)$ cases and $21 \mathrm{TL}$ in only 1 (6.3\%) case. Therefore, to the best of our knowledge, the present study provides the first large data set for future colugo studies. Although our range of vertebral formulae were similar to those of previous reports, our data clearly showed high variation in individuals, with meristic deviation and substantial geographical variation of vertebral formulae between two colugos: 7C, 13-14T, 6-8L, 4-5S, and 19-22TL (mean: 20.9TL) in Philippine colugos and 7C, 13-14T, 5-7L, 4-5S, and 18-20TL (mean: 19.0TL) in Sunda colugos. Although Janečka et al. [25] reported evidence for three species of Sunda colugos based on their skull morphology, distinct vertebral formulae based on geography in the three subspecies of Sunda colugos were not found in the present study. These data strongly indicate that vertebral formulae in colugos reflect selection from gliding adaptation and geographical variation between two species.

In tree shrews, functional adaptation of the vertebral column has been well debated, although most data on vertebral formulae are also surprisingly poor. Most studies that used small sample sizes, mostly one specimen, showed that the average number of vertebrae for Tupaia tree shrews [32, 40], Urogale [40], Dendorogale [13,40], and Anathana [58] was 7C, 13T, 6L, 3S, and 19TL, whereas Ptilocercus tree shrews were 7C, 14T, 5L, 3S, and 19TL [22, 30, 32, 40]. These reports showed consistent vertebral formula for Tupaia species (7C, 13T, 6L, and 3S) and Ptilocercus species (7C, 14T, 5L, and 3S). According to Sánchez-Villagra et al. [42], all eight tree shrews (Ptilocercus lowii) were 19TL. In another studies, Shultz [43] examined axial vertebrae in 16 Tupaia tree shrews. He classified the cases with transitional (asymmetrical) vertebrae as intermediate number as follows: $12.5 \mathrm{~T}(1 / 16,6 \%), 13 \mathrm{~T}(14 / 16,88 \%), 14 \mathrm{~T}$ $(1 / 16,6 \%) ; 5 \mathrm{~L}(1 / 16,6 \%), 6 \mathrm{~L}(13 / 16,82 \%), 6.5 \mathrm{~L}$ $(1 / 16,6 \%), 7 \mathrm{~L}(1 / 16,6 \%) ; 18 \mathrm{TL}(1 / 16,6 \%), 19 \mathrm{TL}$ (13/16, 81\%), 20TL (2/16, 13\%); $3 \mathrm{~S}(15 / 16,94 \%)$, $4 \mathrm{~S}(1 / 16,6 \%)$. 
Based on previous data, Sargis [40] commented that arboreal Ptilocercus had more thoracic and fewer lumbar vertebrae than terrestrial Tupaiines, and the axial vertebral column in Tupaia might be more flexible than that in Ptilocercus; therefore, Tupaia is more adapted to the ground.

Our results using a large sample size of 112 tree shrews showed higher variation in TL counts (19TL in $81 / 112,72.3 \%$ ) than previous data. Unfortunately, we could not confirm whether arboreal Ptilocercus has more thoracic vertebrae than terrestrial Tupaia because of the limited sample size of Ptilocercus in our present study. However, the most frequent vertebral formulae in Urogale tree shrews (predominately terrestrial [15]) was also $7 \mathrm{C}, 14 \mathrm{~T}, 5 \mathrm{~L}$, and $3 \mathrm{~S}(5 / 10,50.0 \%)$. In addition, the typical vertebral formula of $13 \mathrm{~T}, 6 \mathrm{~L}$, and 19TL in more terrestrial Tupaia species was present in only $63 / 99$ (63.7\%) individuals in our study. Therefore, our results did not support clearly the previous idea regarding the vertebral difference between living in arboreal and terrestrial habitats [40].

Rodents are definitely the least variable group without any deviation in thoracolumbar counts as shown in other studies $[2,33,42]$. Our study showed that most arboreal and terrestrial rodents were fixed at 19TL without any deviation, but most flying squirrels showed relatively high variation among populations and frequencies (18-21TL), and most semi-aquatic mammals had some variation in frequency (18-20TL). Furthermore, scalytailed squirrels were ultimately exceptional rodents, and typically had 25TL (17/19, 89.5\%) with variation. We found only one skeleton illustration of a Pel's flying squirrel (Anomalurus pelii) and it appeared to be 16T, $9 \mathrm{~L}$, and 25TL [14]. Any other previous statistical data on the axial vertebrae were always missing Anomalidae data from rodents and therefore concluded that rodents mostly have a fixed 19 TL.

Furthermore, the lineage-specific TL numbers in bats were also uniquely different: flying foxes (18TL in all three) and fruit bats (16TL in 1 and 17TL in 1 of 2). Similar TL modification in bats has been reported (14T4L in Pteropus sp. [38]; 11T5L in Plecotus auritus [34]; 11T5L in Myotis [57]; 13T5L in Rousette [35]). In addition, our finding regarding sugar gliders also showed $18 \mathrm{TL}(2 / 13,15.4 \%)$ among the relatively consistent 19 TL counts in examined marsupials. According to Barbour [3], the vertebral column always has 26 presacral vertebrae with lineagespecific trade-off of thoracic and lumbar vertebrae [36, 37, 50,63 ]. Flower [17] also described similar characteristics for 27 marsupials, including two sugar gliders.
To elucidate mammalian vertebral transformation by different locomotive modes, some researchers have explained the functional aspects: various locomotive behaviours such as suspension, gliding, bridging between branches, climbing, and clinging, are considered to be related to more stable and less flexible vertebral columns in mammals $[5,9,26,40$, $44,60]$. Therefore, detailed lumbar vertebral characteristics and their relationships with the locomotive behaviours have been examined [16, 18, 20, 21].

To understand these relationships correctly, it is important to elucidate by osteological, ligamentous, and/or muscular mechanisms [20]; however, it is difficult to identify their relationships because of the complex nature of these mechanisms. Therefore, we tried to obtain a perspective on the vertebral modification by different locomotive modes based on a large data set of vertebral counts and proportions.

Our vertebral data clearly indicate that some aerodynamic mammals, such as colugos, southern flying squirrels, scaly-tailed squirrels, and bats, show exceptionally high frequencies of variation in thoracic, lumbar, and thoracolumbar counts; and sugar gliders and some semi-aquatic rodents also show some variation. These results indicate that morphological change in vertebral formulae by aerodynamic adaptation is possible as a selection.

\section{Morphological transformation of vertebral proportion among different locomotive modes}

Although the specialised and proportional modifications of the forelimb in some aerodynamic mammals have been well examined [4, 28, 46, 47, 49, 53-55], it is still not fully understood whether vertebral proportions have been modified by aerodynamic adaptation. Thorington and Santana [55] compared the body skeletal proportions among Glaucomys (gliding squirrels) and Microsciurus (non-gliding squirrels), and reported longer forearms and lumbar and mid-caudal vertebrae and shorter hands and feet in gliding squirrels compared with non-gliding squirrels as morphological modifications for evolution of gliding flight. In the present study, we confirmed longer lumbar and shorter thoracic vertebrae in flying squirrels and in scaly-tailed squirrels. These results show a shared trait in gliding rodents, and this elongation might be a unique selection and have only evolved in gliding rodents. Furthermore, Dermoptera and Chiroptera have prominently longer thoracic and shorter lumbar vertebrae, but sugar gliders have almost the 
same thoracic and lumbar vertebral proportions as seen in the other mammals adapted to arboreal, terrestrial, and digging habitats. Therefore, each glider may have a unique vertebral proportion with different developmental constraints. This is a developmental issue that should be further addressed in the future.

Our findings also clearly showed prominently elongated cervical and thoracic vertebral proportions in colugos and bats (Table 4). Jenkins [26] discussed how thickened ribs might facilitate slow climbing and bridging in lorisine primates by increasing the stability of the thorax, which, in turn, increases the stability of the vertebral column. Based on his hypothesis, both colugos and bats seem to share unique functional morphological characteristics. Colugos and bats have wide ribs, and another feature considered to be a synapomorphy uniting these two groups in supraorder Volitantia [45, 46, 47, 51, 52, 61]. Because there are fewer ribs in the tree shrews than in the colugos and bats, the morphological characteristics of the ribs might be associated with functional convergence. The elongated thoracic vertebrae with wide and more ribs have been considered to be advantageous in the chiropterans for hanging $[46,47,51,52]$ and/or for stability of the thorax during flight [48].

Of mammals we examined, colugos and bats have elongated necks, and these are interestingly hanging and roosting species that do not make a nest. Because they always have to pay attention to their predators during roosting, it is beneficial to be equipped with a flexible and rotating neck to search for predators in addition to advantageous for enlargement of the propatagium.

Furthermore, tree sloths are also hanging species but were not examined in this research. Because threetoed sloths (Bradypus sp.) are known to have nine cervical vertebrae, these species may be advantageous for head rotation during roosting and hanging. However, two-toed sloths (Choloepus sp.) imply some difficulties when compared with other mammals in cervical count: $5 \mathrm{CV}$ in $6 / 49$ (12\%), 6CV in $42 / 49$ (86\%), and 7CV in $1 / 49$ $(2 \%)$ in C. hoffmanni; seven cervical vertebrae in almost all C. didactylus [7]. These results regarding specialised elongated and shortened cervical vertebrae may show a relationship with ecological selection.

Therefore, a relatively elongated neck might be considered a contributing factor to the derived functional adaptation of the specialised roosting habits and developed propatagium, and not a flying/gliding adaptation.

\section{CONCLUSIONS}

We tested our hypothesis that aerodynamic adaptation modifies axial vertebrae based on locomotive mode, and produced a large data set of vertebral formulae and proportions for colugos, tree shrews, rodents and others, which included all aerodynamic mammals. Our results show that aerodynamic mammals have high variation in thoracic, lumbar, and TL counts and proportions. Therefore, aerodynamic adaptation is potentially a selection factor that, despite developmental constraints, shapes variation among mammals with different locomotive modes, such as arboreal, terrestrial, digging, and semi-aquatic.

\section{Acknowledgements}

For proving the opportunity to measure dry collections, the authors thank Mr. Darrin P. Lunde, Ms. Esther M. Langan, Ms. Nicole R. Edmison, Dr. Kristofer M. Helgen, Dr. James G. Mead, Mr. Charles W. Potter, Ms. Suzanne C. Peurach, Ms. Linda Gordon, Mr. John J. Ososky, and all staff of the Division of Mammals, National Museum of Natural History, Smithsonian Institution (Washington, DC, USA); Dr. Lawrence Heaney, Mr. William Stanley, and Ms. Tracy Damitz of the Field Museum of Natural History (Chicago, IL, USA); Dr. Nancy B. Simmons and Ms. Eileen Westwig of the Department of Mammalogy, American Museum of Natural History (New York, NY, USA); Ms. Judy Chupasko of the Department of Mammals, Museum of Comparative Zoology, Harvard University (Boston, MA, USA); Dr. Philip Unitt of the Department of Bird and Mammals, San Diego Natural History Museum (San Diego, CA, USA); Dr. Janet K. Braun and Ms. Marcia A. Revelez of the Department of Mammalogy, Sam Noble Oklahoma Museum of Natural History, University of Oklahoma (Norman, OK, USA); Ms. Yen-Jean Chen and staff of the Division of Birds and Mammals, Department of Zoology, National Museum of Natural Science (Taichung, Taiwan); Dr. Yi-Jung Lin of the Museum of Zoology and College of Life Science, National Taiwan University (Taipei, Taiwan); Prof. Kenjiro Matsuno, Prof. Hisashi Ueta, and Prof. Hideo Takahashi of the Department of Anatomy, Dokkyo Medical University (Mibu, Tochigi, Japan); Mr. Wayne Longmore and Dr. Erich Fitzgerald of the Melbourne Museum (Melbourne, VIC, Australia); Mr. Jay Villenarette, Mr. Josh Villenarette, and Mr. Joey Williams of the Museum of Osteology (Oklahoma City, OK, USA); Dr. Robert Asher and Mr. Mathew Lowe of Cambridge Museum of Zoology (Cambridge, UK); 
Dr. Nicolas Violaine and Dr. Guillaume Billet of Muséum National d'Histoire Naturelle (Paris, France); and Dr. Hiroko Hirotani and Dr. Hajime Taru of Kanagawa Prefectural Museum of Natural History (Odawara, Kanagawa, Japan).

This study was supported by Scientific Research Grants (KAKENHI) from the Japanese Ministry of Education, Culture, Sports, Science and Technology (2004-2006, No. 16790804; 2007-2009, No. 19790985; 2011-2014, No. 23791579; 2016-2019, No. 16K10640); a grant from the International Research Programme of the Japanese Ministry of Education, Culture, Sports, Science, and Technology (2008-2009); a Visiting Research Grant from the Field Museum of Natural History (2008-2009); and a grant from the Project research program of Toho University Medical School (H23-29, H24-02, H26-01, H27-4).

\section{REFERENCES}

1. Aimi M. Numerical variation of vertebrae in Japanese macaques, macaca fuscata. Anthropol Sci. 1994; 102(Supplement): 1-10, doi: 10.1537/ase.102.supplement_1.

2. Asher RJ, Lin $\mathrm{KH}$, Kardjilov $\mathrm{N}$, et al. Variability and constraint in the mammalian vertebral column. J Evol Biol. 2011; 24(5): 1080-1090, doi: 10.1111/j.1420-9101.2011.02240.x, indexed in Pubmed: 21338435.

3. Barbour RA. Anatomy of marsupials. In: Stonehouse B and Gilmore D (eds.). The biology of marsupials. University Park Press, Baltimore 1977: 237-272.

4. Beard KC. Origin and evolution of gliding in early Cenozoic Dermoptera (Mammalia, Primatomorpha). In: MacPhee RDE (ed.). Primates and their relatives in phylogenetic perspective. Plenum Press, New York 1993: 63-90.

5. Boyer DM, Bloch Jl. Evaluating the mitten-gliding hypothesis for Paromomyidae and Micromomyidae (Mammalia, "Plesiadapiformes") using comparative functional morphology of new Paleogene skeletons. In: Dagosto MJ, Sargis EJ (eds.). Mammalian evolutionary morphology. A tribute to Frederick S. Szalay. Springer-Verlag, New York 2008: 231-279.

6. Buchholtz E. Vertebral and rib anatomy in Caperea marginata: Implications for evolutionary patterning of the mammalian vertebral column. Marine Mammal Science. 2010; 27(2): 382-397, doi: 10.1111/j.1748-7692.2010.00411.x.

7. Buchholtz EA, Stepien CC. Anatomical transformation in mammals: developmental origin of aberrant cervical anatomy in tree sloths. Evol Dev. 2009; 11(1): 69-79, doi: 10.1111/j.1525142X.2008.00303.x, indexed in Pubmed: 19196334.

8. Burke AC, Nelson CE, Morgan BA, et al. Hox genes and the evolution of vertebrate axial morphology. Development. 1995; 121(2): 333-346, indexed in Pubmed: 7768176.

9. Cartmill M, Milton K. The lorisiform wrist joint and the evolution of "brachiating" adaptations in the hominoidea. Am J Phys Anthropol. 1977; 47(2): 249-272, doi: 10.1002/ ajpa.1330470206, indexed in Pubmed: 410307.

10. Clutton-Brock J, Wilson DE. Mammals. Smithsonian handbooks DK, New York 2002.

11. Cohn MJ, Tickle C. Developmental basis of limblessness and axial patterning in snakes. Nature. 1999; 399(6735): 474-479, doi: 10.1038/20944, indexed in Pubmed: 10365960.
12. Cordes R, Schuster-Gossler K, Serth K, et al. Specification of vertebral identity is coupled to Notch signalling and the segmentation clock. Development. 2004; 131(6): 1221-1233, doi: 10.1242/dev.01030, indexed in Pubmed: 14960495.

13. Davis DD. Notes on the anatomy of the treeshrew Dendrogale. Field Mus Nat His (Zool Series. 1938; 20: 383-404.

14. De Blainville HMD. Ostéographie ou description iconographique comparée du squelette et du système dentaire des mammifères récents et fossils. JB Bailliére et Fils. Paris. 1840.

15. Doyle GA, Walker AC. D'Souza F.A preliminary field report on the lessor tree shrew Tupaia minor. In: Martin RD, Doyle GA, Walker AC (eds.). Prosimian biology. Duckworth, London 1974: 167-182.

16. Filler AG. Homeotic evolution in the mammalia: diversification of therian axial seriation and the morphogenetic basis of human origins. PLoS One. 2007; 2(10): e1019, doi: 10.1371/ journal.pone.0001019, indexed in Pubmed: 17925867.

17. Flower W. An introduction to the osteology of the Mammalia. Macmillan, London. 1885, doi: 10.5962/bhl.title.101537.

18. Galis F, Carrier DR, van Alphen J, et al. Fast running restricts evolutionary change of the vertebral column in mammals. Proc Natl Acad Sci USA. 2014; 111(31): 11401-11406, doi: 10.1073/pnas.1401392111, indexed in Pubmed: 25024205.

19. Gaunt SJ. Conservation in the Hox code during morphological evolution. Int J Dev Biol. 1994; 38(3): 549-552, indexed in Pubmed: 7848839.

20. Granatosky MC, Lemelin P, Chester SGB, et al. Functional and evolutionary aspects of axial stability in euarchontans and other mammals. J Morphol. 2014; 275(3): 313-327, doi: 10.1002/jmor.20216, indexed in Pubmed: 24288155.

21. Granatosky MC, Miller CE, Boyer DM, et al. Lumbar vertebral morphology of flying, gliding, and suspensory mammals: implications for the locomotor behavior of the subfossil lemurs Palaeopropithecus and Babakotia. J Hum Evol. 2014; 75: 40-52, doi: 10.1016/j.jhevol.2014.06.011, indexed in Pubmed: 25216795.

22. Gregory WK. Relationship of the Tupaiidae and of Eocene Lemurs, especially Notharctus. Bull Geol Soc Am. 1913; 24(1): 247-252, doi: 10.1130/gsab-24-247.

23. Hautier L, Weisbecker V, Sánchez-Villagra MR, et al. Skeletal development in sloths and the evolution of mammalian vertebral patterning. Proc Natl Acad Sci U S A. 2010; 107(44): 18903-18908, doi: 10.1073/pnas.1010335107, indexed in Pubmed: 20956304.

24. Ikeya M, Takada S. Wnt-3a is required for somite specification along the anteroposterior axis of the mouse embryo and for regulation of cdx-1 expression. Mech Dev. 2001; 103(1-2): 27-33, indexed in Pubmed: 11335109.

25. Janecka JE, Miller W, Pringle TH, et al. Molecular and genomic data identify the closest living relative of primates. Science. 2007; 318(5851): 792-794, doi: 10.1126/science.1147555, indexed in Pubmed: 17975064.

26. Jenkins FA. Anatomy and function of expanded ribs in certain edentates and primates. J Mammal. 1970; 51(2): 288-301, indexed in Pubmed: 5425659.

27. Kawashima T, Murakami K, Takayanagi M, et al. Evolutionary transformation of the cervicobrachial plexus in the colugo (Cynocephalidae: Dermoptera) with a comparison to treeshrews (Tupaiidae: Scandentia) and strepsirrhines (Strepsirrhini: Primates). Folia Morphol. 2012; 71(4): 228-239, indexed in Pubmed: 23197142.

28. Kawashima T, Thorington RW, Bohaska PW, et al. Evolutionary Transformation of the Palmaris Longus Muscle in Flying Squirrels (Pteromyini: Sciuridae): An Anatomical Consideration of the Origin of the Uniquely Specialized Styliform Cartilage. Anat Rec. 2017; 300(2): 340-352, doi: 10.1002/ar.23471, indexed in Pubmed: 27611816. 
29. Leche W. Über die Säugethiergattung Galeopithecus eine Morphologische Untersuchung. Kungl Svenska Ventenskap Akad Stochholm. 1886; 21: 3-92.

30. Le Gros Clark WE. On the Anatomy of the Pen-tailed TreeShrew (Ptilocercus lowii.). Proc zool Soc Lond. 1926; 96(4): 1179-1309, doi: 10.1111/j.1096-3642.1926.tb02241.x.

31. Lim N. Colugo. The flying lemur of South-east Asia. Draco Publishing \& Distribution Pte Ltd. And National University of Singapore, Singapore 2007.

32. Lyon M. Treeshrews: An account of the mammalian family Tupaiidae. Proc US Nat Mus. 1913; 45(1976): 1-188, doi: 10.5479/si.00963801.45-1976.1.

33. Narita Y, Kuratani S. Evolution of the vertebral formulae in mammals: a perspective on developmental constraints. J Exp Zool B Mol Dev Evol. 2005; 304(2): 91-106, doi: 10.1002/ jez.b.21029, indexed in Pubmed: 15660398.

34. Norberg UM. Functional osteology and myology of the wing of Plecotus auritus Linnaeus (Chiroptera). Arkiv för Zoologi. 1968; 22: 483-543.

35. Norberg U. Functional osteology and myology of the wing of the dog-faced bat Rousettus aegyptiacus (É. Geoffroy) (Mammalia, Chiroptera). Z Morph Tiere. 1972; 73(1): 1-44, doi: $10.1007 /$ bf00418146.

36. Owen R. Outlines of a classification of the Masupialia. Trans Zool Soc London. 1841; 2: 315-333.

37. Owen R. On the osteology of the Marsupialia. Trans Zool Soc London. 1841; 2: 379-408.

38. Owen R. Descriptive catalogue of the osteological series contained in the museum of the of England. Royal College of Surgeons. London. 1853.

39. Pilbeam D. The anthropoid postcranial axial skeleton: comments on development, variation, and evolution. J Exp Zool B Mol Dev Evol. 2004; 302(3): 241-267, doi: 10.1002/jez.b.22, indexed in Pubmed: 15211685.

40. Sargis E. A preliminary qualitative analysis of the axial skeleton of tupaiids (Mammalia, Scandentia): functional morphology and phylogenetic implications. J Zool. 2001; 253(4): 473-483, doi: 10.1017/s0952836901000437.

41. Sawin P. Preliminary studies of hereditary variation in the axial skeleton of the rabbit. Anat Rec. 1937; 69(4): 407-428, doi: 10.1002/ar.1090690403.

42. Sánchez Villagra M, Narita Y, Kuratani S. Thoracolumbar vertebral number: The first skeletal synapomorphy for afrotherian mammals. Systematics and Biodiversity. 2007; 5(1): 1-7, doi: $10.1017 / \mathrm{s} 1477200006002258$.

43. Schultz A. Vertebral column and thorax. In: Hofer H, Schultz AH, Starck D (eds.). Primatologia Vol IV S Karger, Basel 1961: 1-66.

44. Shapiro $\amalg$, Simons CVM. Functional aspects of strepsirrhine lumbar vertebral bodies and spinous processes. J Hum Evol. 2002; 42(6): 753-783, doi: 10.1006/jhev.2002.0560, indexed in Pubmed: 12069508.

45. Shoshani J, McKenna MC. Higher taxonomic relationships among extant mammals based on morphology, with selected comparisons of results from molecular data. Mol Phylogenet Evol. 1998; 9(3): 572-584, doi: 10.1006/mpev.1998.0520, indexed in Pubmed: 9668007.
46. Simmons NB. Bat relationship and origin of flight. Symp Zool Soc Lond. 1995; 67: 27-43.

47. Simmons N, Quinn T. Evolution of the digital tendon locking mechanism in bats and dermopterans: A phylogenetic perspective. J Mammal Evol. 1994; 2(4): 231-254, doi: 10.1007/ bf01464276.

48. Slijper EJ. Comparative biologic-anatomical investigations on the vertebral column and spinal musculature of mammals. Verh K Ned Akad Wet Tweede Sectie. 1946; 42: 1-128.

49. Stafford B, Jr. RT, Kawamichi T. Gliding behavior of japanese giant flying squirrels (Petaurista leucogenys). J Mammal. 2002; 83(2): 553-562, doi: 10.1644/1545-1542(2002)083<0553:gb ojgf>2.0.co;2.

50. Stirling EL. Description of a new genus and species of Marsupialia, 'Notoryctes typhlops'. Trans Roy Soc S Australia. 1891; 14: 154-187.

51. Szalay FS, Lucas SG. ranioskeletal morphology of archontans, and diagnoses of Chiroptera, Vilitantia, and Archonta. In: MacPhee RDE (ed.). Primates and their relatives in phylogenyetic perspective, Advances in Primatology Series. Plenum Press, New York 1993: 187-226.

52. Szalay FS, Lucas SG. Postcranial morphology of Paleocene Chriacus and Mixodectes and the phylogenetic relationships of archontan mammals. New Mexico Bulletin of Natural History and Science. 1996; 7: 1-47.

53. Thorington RW, Darrow K, Anderson CG. Wing Tip Anatomy and Aerodynamics in Flying Squirrels. J Mammal. 1998; 79(1): 245-250, doi: 10.2307/1382860.

54. Thorington RW, Heaney LR. Body Proportions and Gliding Adaptations of Flying Squirrels (Petauristinae). J Mammal. 1981; 62(1): 101-114, doi: 10.2307/1380481.

55. Thorington R, Santana E. How to make a flying squirrel: glaucomysanatomy in phylogenetic perspective. J Mammal. 2007; 88(4): 882-896, doi: 10.1644/06-mamm-s-325r2.1.

56. Varela-Lasheras I, Bakker AJ, van der Mije SD, et al. Breaking evolutionary and pleiotropic constraints in mammals: On sloths, manatees and homeotic mutations. Evodevo. 2011; 2: 11, doi: 10.1186/2041-9139-2-11, indexed in Pubmed: 21548920.

57. Vaughan TA. The skeletal system. In: Wimsatt WA (ed.). Biology of Bats vol. 1. Chapter 3. Academic Press, New York 1970: 97-138.

58. Verma K. Notes on the biology and anatomy of the indian tree-shrew, anathana wroughtoni. Mammalia. 1965; 29(3): 289-330, doi: 10.1515/mamm.1965.29.3.289.

59. Viglino M, Flores DA, Ercoli MD, et al. Patterns of morphological variation of the vertebral column in dolphins. J Zool. 2014; 294(4): 267-277, doi: 10.1111/jzo.12177.

60. Ward C. Torso morphology and locomotion inProconsul nyanzae. Am J Phys Anthropol. 1993; 92(3): 291-328, doi: 10.1002/ ajpa.1330920306.

61. Wible JR, Novacek MJ. Cranial evidence for the monophyletic origin of bats. Am Mus Novitates. 1988; 2911: 1-19.

62. Williams S. Variation in anthropoid vertebral formulae: implications for homology and homoplasy in hominoid evolution. J Exp Zool. 2011: 134-147, doi: 10.1002/jez.b.21451.

63. Wood Jones F. The mammals of South Australia. Govt Printer, Adelaide 1923. 